\title{
CHARACTERIZATIONS OF RIGHT NAKAYAMA RINGS
}

\author{
by MANABU HARADA
}

(Received 21 August, 1990)

We have studied relationships between almost relative projectivity and Nakayama rings [8]. In this paper we shall further investigate certain characterizations of right Nakayama rings in terms of almost relative projectives (or injectives). We shall consider three conditions (A), (B) and (C) (see Section 1), which are always satisfied for the relative projective modules, but not for almost relative projectives in general. As an application of [9, Theorem] and [10, Theorem 2], we shall show that a right artinian ring is right Nakayama if and only if one of the above three conditions holds true for almost relative projectives (Corollary to Theorem 1 ). Moreover we shall give a characterization of two-sided Nakayama rings related to $(C)$ and the dual $\left(C^{\#}\right)$ (Theorem 2). Finally we shall investigate the transitivity of almost relative projectives, which is the converse of $(B)$, and give some characterizations of right Nakayama rings related to the transitivity.

1. Preliminaries. In this paper we always assume that $R$ is an associative ring with identity and every module $M$ is a unitary right $R$-module. We shall denote the length, the socle and the Jacobson radical of $M$ by $|M|$, $\operatorname{Soc}(M)$ and $J(M)$, respectively. In particular we denote $J(R)$ by $J$. We follow [8] and [11] for other terminology. We recall here the definition of almost relative projectivity [8]. Let $M$ and $N$ be $R$-modules. For any diagram with $K$ a submodule of $M$ :

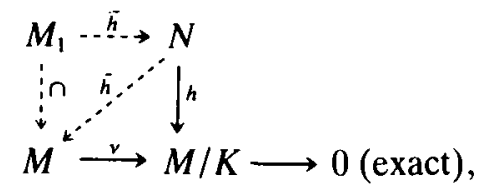

if either there exists $\bar{h}: N \rightarrow M$ with $v \bar{h}=h$ or there exist a non-zero direct summand $M_{1}$ of $M$ and $\bar{h}: M_{1} \rightarrow N$ with $h \bar{h}=v \mid M_{1}$, then $N$ is called almost $M$-projective [8] (if we always obtain the first case, we say that $N$ is $M$-projective [2]).

Let $\left\{M_{i}, N_{j}\right\}_{i=1, j=1}^{m}$ be any set of finitely generated $R$-modules such that $M_{i}$ is almost $N_{j}$-projective for any pair $i$ and $j$. We consider the following property:

(A) $\sum_{i}^{\oplus} M_{i}$ is always almost $\sum_{j}^{\oplus} N_{j}$-projective for any set $\left\{M_{i}, N_{j}\right\}$ as above.

As is easily seen, the above property is equivalent to $M_{i}$ being almost $\sum_{j}^{\oplus} N_{j}$ projective for all $i$ (cf. [5, Lemma 2]). We note that $\Sigma^{\oplus} M_{i}$ is $\Sigma^{\oplus} N_{j}$-projective if $M_{i}$ is $N_{j}$-projective for all $i$ and $j[2]$.

Let $R$ be a perfect ring. Let $M_{0}, M_{1}$ and $M_{2}$ be finitely generated $R$-modules and $M_{1}$ indecomposable. Assume that $M_{0}$ is almost $M_{1}$-projective but not $M_{1}$-projective. Then $M_{1}$ is $M_{2}$-projective by [9, Proposition 1], if $M_{0}$ is $M_{2}$-projective. However if $M_{0}$ is almost $M_{2}$-projective, then $M_{1}$ is not almost $M_{2}$-projective in general.

By (B) we shall denote the above property:

(B) For any indecomposable $R$-module $M_{1}$ and any finitely generated $R$-modules $M_{0}$ 
and $M_{2}$, if $M_{0}$ is almost $M_{1}$-projective but not $M_{1}$-projective, and $M_{0}$ is almost $M_{2}$-projective, then $M_{1}$ is always almost $M_{2}$-projective.

Finally we shall give one more property of relative projectives which is not satisfied for almost relative projectives. Let $M$ and $N$ be $R$-modules. Assume that $M$ is $N$-projective. Then it is well known that $M$ is $N^{\prime}$-projective and $N / N^{\prime}$-projective for any submodule $N^{\prime}$ of $N$. We study the same properties for almost relative projectives. We assume that $M$ is almost $N$-projective. Take a diagram for a submodule $K$ of $N^{\prime}$ :

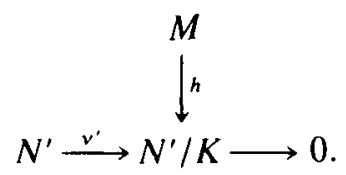

From the above we can derive the diagram:

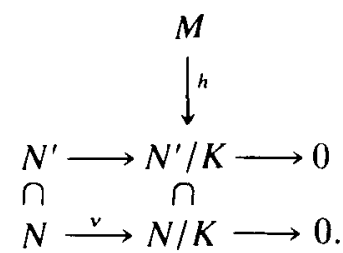

Assume that there exist a non-zero direct summand $N_{1}$ of $N$ and $\tilde{h}: N_{1} \rightarrow M$ such that $h \bar{h}=v \mid N_{1}$. Then $v\left(N_{1}\right)=\left(N_{1}+K\right) / K \subset h \tilde{h}\left(N_{1}\right) \subset h(M) \subset N^{\prime} / K$. Hence $N_{1} \subset N^{\prime}$, because $v$ is the canonical epimorphism. Accordingly $N_{1}$ is also a direct summand of $N^{\prime}$, and $h \bar{h}=v^{\prime} \mid N_{1}$. If there exists $\tilde{h}: M \rightarrow N$ with $v \bar{h}=h$, then $\tilde{h}(M) \subset N^{\prime}$ as above. Therefore $M$ is almost $N^{\prime}$-projective. However $M$ is not almost $N / N^{\prime}$-projective in general. Hence we consider the following property:

(C) If $M$ is almost $N$-projective, then $M$ is also almost $N / N^{\prime}$-projective for any submodule $N^{\prime}$ of $N$.

Related to (C) we can consider the following condition: If $M$ is almost $N$-projective, then $M^{\prime}$ (or $M / M^{\prime}$ ) is also almost $N$-projective, where $M^{\prime}$ is any submodule of $M$. We shall study this property in the forthcoming paper [12].

2. Right Nakayama rings. We assume that $R$ is right artinian. If $e R$ is a uniserial module for each primitive idempotent $e$, we call $R$ a right Nakayama ring. We shall give several characterizations of right Nakayama rings with respect to the above properties (A), (B) and (C). We recall here the definitions of LPSM and lifting modules ([9] and [13]). Let $\left\{e_{i}\right\}_{i=1}^{n}$ be a set of primitive idempotents and $A_{i}$ a submodule of $e_{i} R$ for each $i$. If every element $f$ in $\operatorname{Hom}_{R}\left(e_{i} R / e_{j} J, e_{j} R / e_{j} J\right)$ for any pair $(i, j)$ (or $f^{-1}$ ) is lifted to an element in $\operatorname{Hom}_{R}\left(e_{i} R / A_{i}, e_{j} R / A_{j}\right.$ ) (or in $\operatorname{Hom}_{R}\left(e_{j} R / A_{j}, e_{i} R / A_{i}\right)$ ), then we say that $\sum_{i}^{\oplus} e_{i} R / A_{i}$ has the lifting property of simple modules, briefly LPSM. Next, let $M$ be an $R$-module. If, for any submodule $N$ of $M$, there exists a direct decomposition $M=M_{1} \oplus M_{2}$ such that $N \supset M_{1}$ and $N \cap M_{2}$ is a small submodule of $M_{2}$, then we call $M$ a lifting module. 
We note that if $e_{1} R / A_{1}$ is almost $e_{2} R / A_{2}$-projective or $e_{1} R \neq e_{2} R$, then $e_{1} R / A_{1} \oplus e_{2} R / A_{2}$ has LPSM by definition.

We frequently use the following.

Lemma 1([6, Theorem 5]). Assume that $R$ is a semi-perfect ring. Let $A_{1}$ and $A_{2}$ be submodules of $e R$ such that $\mathrm{eJ}^{i+1} \subset A_{j} \subset e J^{i}$ for $j=1$, 2. If $e R / A_{1} \oplus e R / A_{2}$ has $\mathrm{LPSM}$, then $A_{1} \subset A_{2}$ or $A_{2} \subset A_{1}$. In particular $R$ is a right Nakayama ring if and only if $R$ is right artinian and every (two) finite direct sum of local modules eR/A has LPSM for each primitive idempotent $e$.

Let $M_{1}$ be indecomposable. If $M_{0}$ is almost $M_{1}$-projective and $M_{1}$ is not a local module, then $M_{0}$ is $M_{1}$-projective by [11, Theorem 1]. Furthermore if a local module $e R / A$ is almost $f R / B$-projective $(e R \neq f R)$, then $e R / A$ is $f R / B$-projective by definition. On the other hand (A), (B) and (C) always hold for relative projectives. From this observation we may study essentially $(\mathrm{A}),(\mathrm{B})$ and $(\mathrm{C})$ on local modules $e R / A$ for a fixed primitive idempotent $\boldsymbol{e}$.

THEOREM 1. Let $R$ be a perfect ring and $e$ a fixed primitive idempotent. Then the following conditions are equivalent:

(1) $e R$ is a uniserial module (and hence $|e R|<\infty$ ),

(2) (A) holds on local modules $e R / A$,

(3) (B) holds whenever $M_{0}, M_{1}$ and $M_{2}$ are local modules of the form $e R / A$,

(4) (C) holds on local modules of the form $e R / A$.

Proof. We first remark that $|e R|<\infty$ if $e R$ is uniserial. Since $R$ is perfect, $e R \supset e J \supset e J^{2} \supset \cdots$ is a composition series of $e R$. Then $e J^{i}=a_{i} R$ for some $a_{i}$ in $e J^{i}-e J^{i+1}$ and $a_{i+1} R=e J^{i+1}=a_{i} J$, i.e., $a_{i+1}=a_{i} j_{i}$ for some $j_{i} \in J$. Therefore $e J^{n}=0$ for some $n$.

$(1) \Rightarrow(2)$. Assume that $e R / A_{0}$ is almost $e R / A_{i}$-projective for $1 \leqq i \leqq m$. Since $e R / A_{i} \oplus e R / A_{j}$ has LPSM for $i \neq j$ by (1) and Lemma $1, e R / A_{0}$ is almost $\Sigma^{\oplus} e R / A_{i^{-}}$ projective by [9, Theorem ].

$(2) \Rightarrow(1)$. Let $A_{1}$ and $A_{2}$ be the modules in Lemma 1 and $e J^{i} \neq 0$. Since $e J^{i}$ is characteristic and $e J e e J^{i} \subset e J^{i+1} \subset A_{j} \subset e J^{i}, e R / e J^{i}$ and $e R / A_{k}$ are mutually almost relative projective for $k=1,2$ by [5, Proposition 2], but $e R / e J^{i}$ is neither $e R / A_{1}$ nor $e R / A_{2^{-}}$ projective by $\left[1\right.$, p. 22 , Exercise 4]. Then $e R / e J^{i}$ being almost $e R / A_{1} \oplus e R / A_{2}$-projective by (2), $e R / A_{1} \oplus e R / A_{2}$ has LPSM by [9, Theorem]. Hence $A_{1} \subset A_{2}$ or $A_{2} \subset A_{1}$ by Lemma 1 . As a consequence $e J^{i} / e J^{i+1}$ is simple, and so $e R$ is uniserial.

(3) $\Rightarrow(1)$. Let $A_{j}$ be as above. Then $e R / e J^{i}$ is almost $e R / A_{j}$-projective, but not $e R / A_{j}$-projective as in (2) $\Rightarrow(1)$ for $j=1,2$. Hence $e R / A_{1}$ is almost $e R / A_{2}$-projective by (3), and so $e R / A_{1} \oplus e R / A_{2}$ has LPSM from the remark after the definition of LPSM. Therefore $e R$ is uniserial as above.

$(1) \Rightarrow(3)$. Let $B_{0}, B_{1}$ and $B_{2}$ be submodules of $e R$. Assume that $e R / B_{0}$ is almost $e R / B_{1}$-projective, but not $e R / B_{1}$-projective. Then $B_{0} \supset B_{1}$ by (1). If $e R / B_{0}$ is almost $e R / B_{2}$-projective, then $e J e B_{0} \subset B_{2}$ by [11, Proposition 2], and hence $e J e B_{1} \subset e J e B_{0} \subset B_{2}$. Therefore $e R / B_{1}$ is almost $e R / B_{2}$-projective by [11, Proposition 2].

$(1) \Rightarrow(4)$. Assume that $e R$ is uniserial and $M(=e R / A)$ is almost $N(=e R / B)$ projective. Then $e J e A \subset B$. Let $N^{\prime}$ be a submodule of $N$ and $N / N^{\prime}=e R / C$. Since $C \supset B$, $C \supset e J e A$. Further $e R / C \oplus e R / A$ has LPSM by assumption. Therefore $M$ is almost $N / N^{\prime}$-projective by [11, Proposition 2]. 
(4) $\Rightarrow(1)$. Let $A_{i}$ be as in (2) $\Rightarrow(1)$. Then as is shown in (2) $\Rightarrow(1), e R / A_{1}$ is almost $e R / e J^{i+1}$-projective. Hence $e R / A_{1}$ is almost $e R / A_{2}$-projective by (4). Accordingly $e R$ is uniserial as before.

COROLlaRY. Let $R$ be a right artinian ring. Then the following are equivalent:

(1) $R$ is right Nakayama,

(2) (A) holds,

(3) (B) holds,

(4) (C) holds for local modules $M$ and $N$.

Proof. (1) $\Rightarrow(2)$. Let $R$ be right Nakayama. Let $M_{0}, M_{1}$ and $M_{2}$ be finitely generated $R$-modules. Assume that $M_{0}$ is almost $M_{i}$-projective for $i=1,2$. We take a direct decomposition of $M_{i}$ into indecomposable modules $T_{i j}(i=1,2 ; j=1, \ldots, n(i))$. Then $M_{0}$ is clearly almost $T_{i j}$-projective for all $i, j$. We may assume that there exists an integer $m(i)$ such that $M_{0}$ is almost $T_{i k}$-projective but not $T_{i k}$-projective for all $k \geqq m(i)$ and $M_{0}$ is $T_{i k^{\prime}}$-projective for all $k^{\prime}<m(i)(i=1,2)$. Then $T_{i k}$ is a local module by [11, Theorem 1] for $k \geqq m(i)$. Further, $\sum_{\substack{i=1,2 \\ k \geqq m(i)}}^{T_{i k}}$ has LPSM by Lemma 1 and the remark after the definition of LPSM. Hence $M_{0}$ is almost $M_{1} \oplus M_{2}$-projective by [9, Theorem].

(2) $\Rightarrow(3)$. We may assume by [11, Theorem 1] that $M_{1}$ is a local module. Let $M_{2}=\Sigma^{\oplus} T_{2 i}$ be the direct decomposition of $M_{2}$ as in (1) $\Rightarrow(2)$. Then there exists an integer $m$ such that $M_{0}$ is $T_{2 j}$-projective for all $j<m$ and $M_{0}$ is almost $T_{2 j}$-projective but not $T_{2 j^{\prime}}$-projective for all $j^{\prime} \geqq m$. Further since $M_{0}$ is almost $M_{1} \oplus \underset{j^{\prime} \geqq m}{\sum^{\oplus}} T_{2 j^{\prime}}$-projective by (2) and $M_{0}$ is not $M_{1}$-projective, $M_{1} \oplus \sum_{j^{\prime} \geq m}^{\oplus} T_{2 j^{\prime}}$ is a lifting module by [9, Theorem]. Hence $M_{1}$ is almost $\sum_{j^{\prime} \geqq m}^{\oplus} T_{2 j^{\prime}}$-projective by [10, Theorem 1]. Moreover $M_{1}$ is $T_{2 j}$-projective for $j<m$ by [9, Proposition 1]. Hence $M_{1}$ is almost $M_{2}$-projective by (2).

The remaining implications are clear from Theorem 1 and the observation before Theorem 1.

From the above we know that (A) is equivalent to

(A') (A) holds for local modules $M_{i}$ and $N_{j}$

and $(B)$ is equivalent to

(B') (B) holds for local modules $M_{0}, M_{1}$ and $M_{2}$.

However we do not have the same result for (C) (see Theorem 2 below).

Next we shall study a dual result to Theorem 1 . If every indecomposable injective module is uniserial, we say that $R$ is right co-Nakayama ([3] and [6]). We shall give some characterizations of right co-Nakayama rings, which are dual to the Corollary. We refer to [4] for the definition of almost relative injectives. First we define properties $\left(A^{\#}\right),\left(B^{\#}\right)$ and $\left(C^{\#}\right)$ dual to $(A),(B)$ and $(C)$, respectively.

Let $U_{0}, U_{1}$ and $U_{2}$ be finitely generated $R$-modules.

$\left(\mathrm{A}^{\#}\right)$ If $U_{0}$ is almost $U_{i}$-injective for $i=1,2$, then $U_{0}$ is almost $U_{1} \oplus U_{2}$-injective.

Assume that $U_{1}$ is indecomposable. 
$\left(\mathrm{B}^{\#}\right)$ If $U_{0}$ is almost $U_{1}$-injective but not $U_{1}$-injective, and $U_{0}$ is almost $U_{2}$-injective, then $U_{1}$ is always almost $U_{2}$-injective.

$\left(\mathrm{C}^{\#}\right)$ If $U_{0}$ is almost $U_{2}$-injective, then $U_{0}$ is always almost $U^{\prime}$-injective for any submodule $U^{\prime}$ of $U_{2}$.

As the dual to the Corollary we obtain together with results in [10]

COROLlaRY ${ }^{*}$. Let $R$ be a right artinian ring. Then the following conditions are equivalent:

(1) $R$ is right co-Nakayama,

(2) $\left(\mathrm{A}^{\#}\right)$ holds,

(3) $\left(\mathrm{B}^{\#}\right)$ holds,

(4) $\left(\mathrm{C}^{\#)}\right.$ holds whenever $U_{0}$ and $U_{2}$ are finitely generated and uniform.

The implications $(1) \Leftrightarrow(2)$ are given in [4].

Finally we shall give a characterization of two-sided Nakayama rings. Let $\left\{M_{i}\right\}_{i=1}^{n}$ be a set of indecomposable $R$-modules and $M=\sum_{i}^{\oplus} M_{i}$. Take a submodule $N$ of $M$. If there exists a suitable direct decomposition $M=\sum_{i}^{\oplus} M_{i}^{\prime}$ such that $M_{i} \approx M_{i}^{\prime}$ for all $i$ and $N=\sum_{i}^{\oplus} N \cap M_{i}^{\prime}$, then we call $N$ a standard submodule. The following lemma is well known.

Lemma 2. Let $R$ be a two-sided Nakayama ring. Then any submodule $T$ of $P=\sum_{i=1}^{n} e_{i} R$ is a standard submodule, where the $e_{i}$ are primitive idempotents.

Proof. See [15], [16, Section 55] or [8, Lemma 5].

If $e R e$ is a local ring for an idempotent $e, e$ is called a local idempotent. In this case $e J$ is the unique maximal submodule in $e R$.

Lemma 3. Let $R$ be any ring and $e_{1}, e_{2}$ local idempotents. Let $B$ be a submodule in $e_{1} J \oplus e_{2} J \subset e_{1} R \oplus e_{2} R$ and $C$ a submodule in $e_{1} J$ such that $B \not C \oplus 0$. If $e_{1} R / C$ is almost $\left(e_{1} R \oplus e_{2} R\right) / B$-projective, then $\left(e_{1} R \oplus e_{2} R\right) / B$ is decomposable.

Proof. Since $M=\left(e_{1} R \oplus e_{2} R\right) / B$ is not local, $e_{1} R / C$ is $M$-projective by [11, Theorem 1], provided $M$ is indecomposable. However since $e_{1} R$ is a projective cover of $e_{1} R / C$, and further there exists a natural homomorphism $h$ of $e_{1} R$ into $M$ such that $h(C) \neq 0$ by assumption, $e_{1} R / C$ is not $M$-projective by $[1, \mathrm{p} .22$, Exercise 4$]$ (cf. the proof of [5, Lemma 6]). Therefore $M$ is decomposable.

From [1, Proposition 2.5] and the dual result to [11, Theorem 1] we obtain dually to the above:

LEMMA $3^{*}$. Let $U_{1}$ and $U_{2}$ be indecomposable injective modules and $B$ an essential submodule of $U_{1} \oplus U_{2}$. If $A$ is almost $B$-injective for $0 \neq A \subset U_{1}$ such that $\pi_{1}(B) \notin A$, then $B$ is decomposable, where $\pi_{1}: U_{1} \oplus U_{2} \rightarrow U_{1}$ is the projection.

Let $e$ be a local idempotent. By $M(e)$ we denote the set of finitely generated $R$-modules $M$ such that $M / J(M)=(e R / e J)^{(n(M))}$, the direct sum of $n(M)$-copies of $e R / e J$. 
THeOREM 2. Let $R$ be a perfect ring and $e$ a fixed primitive idempotent. Then the following conditions are equivalent:

(1) $e R$ is uniserial (and hence $|e R|<\infty$ ) and every submodule in $e R \oplus e R$ is standard,

(2) (C) holds whenever $M$ and $N$ are any members in $\boldsymbol{M}(e)$.

Compare [7, Theorem 5].

Proof. (1) $\Rightarrow(2)$. In this proof $A, A_{j}$ and $A_{j}^{\prime}$ mean submodules of $e R . N$ is a direct sum of local modules, $N=\Sigma^{\oplus} e R / A_{j}$ by [8, Lemma 5]. Assume $M=M_{1} \oplus M_{2}$. Then it is clear from the definition that $M$ is almost $N$-projective if and only if $M_{i}$ is so for $i=1,2$. Hence we may assume $M=e R / A$. Now $N / N^{\prime} \approx \Sigma^{\oplus} e R / A_{j}^{\prime}$ by [8, Lemma 5] for a submodule $N^{\prime}$ of $N$. Then each $e R / A_{j}^{\prime}$ is an epimorphic image of some $e R / A_{i}$. Hence we can assume that any $A_{j}^{\prime}$ contains some $A_{i}$. Since $A_{i} \supset e J e A$ by [11, Proposition 2], $M$ is almost $e R / A_{j}^{\prime}$-projective by the same proposition. As a consequence $M$ is almost $N / N^{\prime}$-projective by Theorem 1 .

(2) $\Rightarrow(1)$. Put $P=e R \oplus e R$ and take submodules $A_{1} \subset B_{1}, A_{2} \subset B_{2}$ in $e R$ such that $h: B_{1} / A_{1} \approx B_{2} / A_{2}$. We shall show that $h$ (or $h^{-1}$ ) is induced from an element in $e R e=\operatorname{Hom}_{R}(e R, e R)$. If $B_{i}=e R$ for $i=1$ or 2 , then this is clear. Hence we assume $B_{i} \subset e J$ for $i=1,2$. Since $e R$ is a uniserial module of finite length by Theorem 1, we may assume $A_{1}=e J^{n_{1}}, B_{1}=e J^{n_{1}-a}, A_{2}=e J^{n_{2}}$ and $B_{2}=e J^{n_{2}-a}\left(n_{1} \geqq n_{2}\right)$. Then $e R / e J^{n_{1}-1}$ is almost $e R / e J^{n_{1}}$-projective and almost $e R / e J^{n_{2}}$-projective by [11, Proposition 2]. Hence $e R / e J^{n_{1}-1}$ is almost $e R / e J^{n_{1}} \oplus e R / e J^{n_{2}}$-projective by Theorem 1 . Put $C=\left(B_{1} / A_{1}\right)(h)$, the graph in $e R e / J^{n_{1}} \oplus e R / e J^{n_{2}}$. Let $\tilde{C}$ be the submodule of $P$ such that $\bar{C} \supset e J^{n_{1}} \oplus e J^{n_{2}}$ and $C=\tilde{C} /\left(e J^{n_{2}} \oplus e J^{n_{2}}\right)$. Then $e R / e J^{n_{1}-1}$ is almost $\left(P /\left(e J^{n_{1}} \oplus e J^{n_{2}}\right)\right) / C=P / \tilde{C}(=\bar{P})$-projective by (2). Since $B_{i} \subset e J, \bar{P}$ is decomposable by Lemma 3 . Hence $h$ is liftable to an element $\tilde{h}$ in $\operatorname{Hom}_{R}\left(e R / e J^{n_{1}}, e R / e J^{n_{2}}\right)$ or in $\operatorname{Hom}_{R}\left(e R / e J^{n_{2}}, e R / e J^{n_{1}}\right)$ by [14, Lemma 2.1] (cf. [7, p. 526, Remark]). $\bar{h}$ is clearly liftable to an element in $e R e$. Therefore we obtain (1) by [8, Lemma 5].

COROllary. Let $R$ be a two-sided artinian ring. Then the following are equivalent:

(1) $R$ is two-sided Nakayama,

(2) (C) holds whenever $M$ and $N$ are finitely generated $R$-modules,

(3) $\left(C^{\sharp}\right)$ holds whenever $U_{0}$ and $U_{2}$ are finitely generated $R$-modules.

Proof. (1) $\Rightarrow(2)$. Assume that $R$ is two-sided Nakayama. Then every finitely generated $R$-module $N$ is a direct sum of local modules, $N=\Sigma^{\oplus} e_{j} R / A_{j}$ (by Lemma 2). Hence we can use the same argument as in the proof of Theorem 2.

$(2) \Rightarrow(1)$. We assume (C) for any finitely generated modules $M$ and $N$. Then $R$ is right Nakayama from the Corollary to Theorem 1. We may assume that $R$ is a basic ring with $J^{2}=0$. Then $e J$ is simple or zero for any primitive idempotent $e$. Assume $h: e_{1} J \approx e_{2} J$ for two primitive idempotents $e_{1}$ and $e_{2}$. Then in the same manner as in the proof of Theorem 2 we can show that $h$ is liftable to an element in $e_{1} R e_{2}$ or in $e_{2} R e_{1}$. Hence $R$ is left Nakayama by [14, Lemma 4.3].

(1) $\Rightarrow(3)$. If $R$ is two-sided Nakayama, then every finitely generated $R$-module is a direct sum of uniserial modules. Hence $R$ is right co-Nakayama and we may assume that $U_{0}$ is uniform and $U_{2}=\sum_{i}^{\oplus} V_{i}$; the $V_{i}$ are unform. Since $U_{0}$ is almost $U_{2}$-injective, $U_{0}$ is almost $V_{i}$-injective for all $i$. Let $U^{\prime}$ be any submodule of $U_{2}$ and $U^{\prime}=\sum_{j}^{\oplus} W_{j}$; the $W_{j}$ are 
uniform. Then every $W_{j}$ is monomorphic to some $V_{i}$, since $W_{j}$ is uniform, and hence $U_{0}$ is almost $W_{j}$-injective from Corollary ${ }^{\#}$. Therefore $U_{0}$ is almost $U^{\prime}$-injective from Corollary $((1) \Rightarrow(2))$. Hence $\left(C^{\#}\right)$ holds.

(3) $\Rightarrow(1)$. We assume $\left(\mathrm{C}^{*}\right)$ for any finitely generated $R$-modules $U_{0}$ and $U$. Then $R$ is right co-Nakayama from Corollary ${ }^{\#}$. In order to show that $R$ is two-sided Nakayama, we may assume $J^{2}=0$. Let $e$ be any primitive idempotent. We shall show that $e R$ is uniserial i.e., $e J$ is simple, provided $e J \neq 0$. Assume $e J \neq 0$ and $e J=A_{1} \oplus A_{2} \oplus B$, where $A_{1}$ is simple, so that $A_{1} \neq 0$, and similarly for $A_{2}$. Put $U_{1}=e R /\left(A_{2} \oplus B\right), U_{2}=e R /\left(A_{1} \oplus B\right)$ and $U=U_{1} \oplus U_{2}$. Then $U_{i}$ is a uniserial module with $\left|U_{i}\right|=2$ for $i=1,2$. Since $R$ is a right co-Nakayama ring with $J^{2}=0, U_{i}$ is injective. Put $U_{0}=A_{1}$ and $E=E\left(U_{0}\right)\left(=U_{1}\right)$. Since $\left|U_{i}\right|=2$ and $U_{i}$ is injective, $U_{0}$ is almost $U_{i}$-injective, by [5, Proposition 5]. As a consequence $U_{0}$ is aimost $U$-injective from Corollary ${ }^{\#}$. We take the submodule $U^{\prime}$ of $U$ such that (i) $U^{\prime} \supset J(U)=J\left(U_{1}\right) \oplus J\left(U_{2}\right), \quad$ (ii) $\quad U^{\prime} / J(U)=\{\bar{x}+\bar{x} \mid \bar{x} \in e R / e J\} \subset$ $e R / e J \oplus e R / e J=U / J(U)$. Then $U_{0}$ is almost $U^{\prime}$-injective by $\left(C^{\#}\right)$. Hence $U^{\prime}$ is decomposable by (ii) and Lemma $3^{\#}$. Since $\left|\operatorname{Soc}\left(U^{\prime}\right)\right|=2$ and $\left|U^{\prime}\right|=3, U^{\prime}=W_{1} \oplus W_{2}$ with $\left|W_{1}\right|=2,\left|W_{2}\right|=1$. From (i) and (ii) we know that $W_{1}$ is uniserial and $\pi_{i} \mid W_{1}$ is an isomorphism, where $\pi_{i}: U \rightarrow U_{i}$ is the projection for $i=1,2$. Hence $U_{1}$ is isomorphic to $U_{2}$ by $t=\left(\pi_{2} \mid W_{1}\right)\left(\pi_{1} \mid W_{1}\right)^{-1}$, which induces the identity mapping of $e R / e J$ by (ii). Therefore there exists $j$ in $e J e$ such that the left sided multiplication of $(e+j)$ gives $t$, i.e., $(e+j)\left(A_{2} \oplus B\right)=A_{1} \oplus B$. Thus $A_{1} \oplus B=A_{2} \oplus B$ for $J^{2}=0$. As a consequence $e J$ is simple, i.e., $R$ is right Nakayama. Therefore $R$ is two-sided Nakayama by [3, Theorem 5.4].

We shall give a right Nakayama ring where (B) does not hold if $M_{1}$ is not indecomposable. Let $R=\sum_{i}^{\oplus} e_{i} R$ be a right Nakayama ring with the following structure $\left(\left\{e_{i}\right\}_{i=1}^{4}\right.$ is a set of mutually orthogonal primitive idempotents with $\left.1=\sum e_{i}\right)$ :

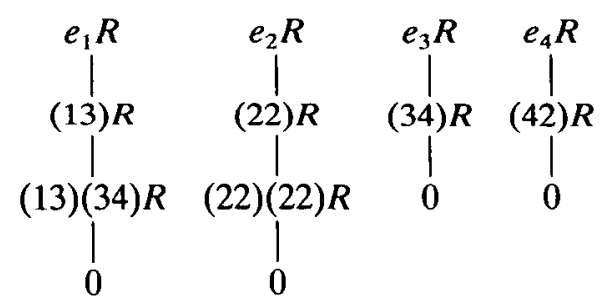

and $R=\Sigma^{\oplus} e_{i} K \oplus \Sigma^{\oplus}(i j) K \oplus \Sigma^{\oplus}(i j)(j k) K,(i j)=e_{i}(i j) e_{j}$ and other products among (ij) are zero except as in the above diagram, where $K$ is a field. Put $A_{0}=(13) R$, $A_{1}=(13)(34) R, B_{0}=(22)(22) R$ and $B_{1}=(22) R$. Then $M_{0}=e_{1} R / A_{0} \oplus e_{2} R / B_{0}$ is almost $M_{1}$ $\left(=e_{1} R / A_{1} \oplus e_{2} R / B_{1}\right)$-projective, but not $M_{1}$-projective and $M_{0}$ is almost $M_{2}\left(=e_{2} R\right)$ projective. However $M_{1}$ is not almost $M_{2}$-projective, since $(22)(22) \neq 0$. Similarly $M_{0}^{\prime}$ (= $\left.e_{1} R / A_{0}\right)$ is almost $M_{1}$-projective, but not $M_{1}$-projective and $M_{0}^{\prime}$ is $M_{2}$-projective. However $M_{1}$ is not $M_{2}$-projective (cf. [9, Proposition 1]).

3. Transitivity on relative projectives. In this section we shall investigate the transitivity of relative projectives: if $M_{0}$ is $M_{1}$-projective and $M_{1}$ is $M_{2}$-projective, is $M_{0}$ then $M_{2}$-projective? The similar property on almost relative projectives is in some sense the converse of $(B)$. 
Proposition 1. Let $R$ be a perfect ring. Then transitivity of relative projectives on $M(e)$ always holds. If transitivity on $\boldsymbol{M}\left(e_{1}\right) \cup \boldsymbol{M}\left(e_{2}\right)$ holds, then $e_{1} J=e_{2} J=0$, where $e_{1} R \approx e_{2} R$.

Proof. Let $M_{1}, M_{2}$ be in $M(e)$. Assume that $M_{1}$ is $M_{2}$-projective and take a projective cover $P_{i}$ of $M_{i}, i=1,2: P_{i}=\sum_{j=1}^{n_{i}} e R_{i j} ; e R_{i j}=e R$, and $M_{i}=P_{i} / A_{i}$. Let $f$ be an element in $\operatorname{Hom}_{R}\left(P_{1}, P_{2}\right) ; f=\sum r_{i j} e_{i j}\left(P_{1} \rightarrow P_{2}\right)$, where $e_{i j}\left(P_{1} \rightarrow P_{2}\right)=1_{e R}: e R_{1 i} \rightarrow e R_{2 j}, r_{i j} \in e R e$. Since $r_{i j} e_{i j} \in \operatorname{Hom}_{R}\left(P_{1}, P_{2}\right), \quad\left(r_{i j} e_{i j}\right) A_{1} \subset A_{2}$ by $\left[1\right.$, p. 22, Exercise 4]. Assume that $M_{2}$ is $M_{3}$-projective and $P_{3}$ is a projective cover of $M_{3}$ as above. Take any element $g$ in $\operatorname{Hom}_{R}\left(P_{1}, P_{3}\right)$ and $g=\left(g_{i j}\right)$. Then $e_{i j}\left(P_{1} \rightarrow P_{3}\right)=e_{i j}\left(P_{2} \rightarrow P_{3}\right) e_{i 1}\left(P_{1} \rightarrow P_{2}\right)$ and $g_{i j} e_{i j}\left(P_{1} \rightarrow\right.$ $\left.P_{3}\right) A_{1}=g_{i j} e_{1 j}\left(P_{2} \rightarrow P_{3}\right) e_{i 1}\left(P_{1} \rightarrow P_{2}\right) A_{1} \subset g_{i j} e_{1 j}\left(P_{2} \rightarrow P_{3}\right) A_{2} \subset A_{3}$. Hence $g\left(A_{1}\right) \subset A_{3}$. Accordingly we obtain the transitivity. Next we assume the second condition in the proposition. Here $e_{1} R / e_{1} J$ is $e_{2} R / e_{2} J^{2}$-projective and $e_{2} R / e_{2} J^{2}$ is $e_{1} R / e_{1} J^{3}$-projective. Hence $e_{1} R / e_{1} J$ is $e_{1} R / e_{1} J^{3}$-projective by the transitivity. Therefore $e_{1} J=e_{1} J^{2}$, and hence $e_{1} J=0$.

From Proposition 1 we have

THEOREM 3. Let $R$ be a perfect ring. Then transitivity of relative projectives over finitely generated modules holds if and only if either $R$ is semi-simple or $R / J(R)$ is a simple ring.

We shall study the above problem for almost relative projectives. In this case the transitivity is the converse of (B). Let $M_{0}, M_{1}$ and $M_{2}$ be finitely generated $R$-modules. The property of transitivity of almost relative projectives is

$\left(\mathrm{B}_{1}\right)$ If $M_{0}$ is almost $M_{1}$-projective and $M_{1}$ is almost $M_{2}$-projective, then $M_{0}$ is always almost $M_{2}$-projective.

Lemma 4. Let $R$ be any ring and $\left\{e_{i}\right\}_{i=1}^{m}$ a set of local idempotents. Put $P=\sum_{i=1}^{m} e_{i} R$ and $\bar{P}=\Sigma^{\oplus} e_{i} R / e_{i} J^{n}$ for a fuxed integer $n$. Assume that every submodule of $\bar{P}$ is standard. Then every submodule $A$ of $P$ which contains $\Sigma^{\oplus} e_{i} J^{n}$ is also standard in $P$.

Proof. Put $\bar{e}_{i} \bar{R}=e_{i} R / e_{i} J^{n}$ and $\bar{A}=A /\left(\sum^{\oplus} e_{i} J^{n}\right)$. Then there exists a direct decomposition of $\bar{P}:=\Sigma^{\oplus} \bar{P}_{i}$ such that $\bar{A}=\sum^{\oplus}\left(\bar{P}_{i} \cap \bar{A}\right)$ and $\bar{P}_{i} \approx \bar{e}_{i} \bar{R}$. Since $\operatorname{End}_{R}\left(\bar{e}_{i} \bar{R}\right)$ is a local ring, we may assume that $\bar{P}_{1}=\bar{e}_{1} \bar{R}\left(\bar{g}_{1}\right)$, where $\bar{g}_{1}: \bar{e}_{1} \bar{R} \rightarrow \sum_{j \neq 1}^{\oplus} \bar{e}_{j} \bar{R}$. Then

$$
\bar{P}=\bar{P}_{1} \oplus \bar{e}_{2} \bar{R} \oplus \cdots \oplus \bar{e}_{m} \bar{R}=\bar{P}_{1} \oplus \bar{P}_{2} \oplus \cdots \oplus \bar{P}_{m}
$$

Considering the projection of $\bar{P}$ onto $\sum_{j \geqslant 2}^{\oplus} \bar{e}_{j} \bar{R}$ in the above we may assume that $\bar{P}_{2}=\bar{e}_{2} \bar{R}\left(\bar{g}_{2}\right)$ with $\bar{g}_{2}: \bar{e}_{2} \bar{R} \rightarrow \bar{P}_{1} \oplus \sum_{k \geqslant 3}^{\oplus} \bar{e}_{k} \bar{R}$. Hence we obtain inductively $\bar{g}_{i}: \bar{e}_{i} \bar{R} \rightarrow \sum_{k<i}^{\oplus} \bar{P}_{k} \oplus \sum_{k^{\prime}>i}^{\oplus} \bar{e}_{k^{\prime}} \bar{R}$ such that $\bar{P}_{i}=\bar{e}_{i} \bar{R}\left(\bar{g}_{i}\right)$ and $\bar{P}=\sum_{k<i}^{\oplus} \bar{P}_{k} \oplus \sum_{k^{\prime}>i}^{\oplus} \bar{e}_{k^{\prime}} \bar{R}$. By induction we can show that $\bar{g}_{i}$ is liftable to an element $g_{i}: e_{i} R \rightarrow \sum_{k \leqq i}^{\oplus} e_{k} R\left(g_{k}\right) \oplus \sum_{k^{\prime}>i}^{\oplus} e_{k^{\prime}} R$ and $P=\sum_{k \leqq i}^{\oplus} e_{i} R\left(g_{i}\right)^{\oplus} \sum_{k^{\prime}>i}^{\oplus} e_{k^{\prime}} R$. Therefore 
we have $P=\sum_{i=1}^{m} e_{i} R\left(g_{i}\right)$ and $\bar{e}_{i} \bar{R}\left(g_{i}\right)=\bar{P}_{i}$ for all $i$. Since $A \supset \Sigma^{\oplus} e_{j} J^{n}=\Sigma^{\oplus} e_{i} R\left(g_{i}\right) J^{n}$, $A \supset \Sigma^{\oplus}\left(e_{i} R\left(g_{i}\right) \cap A\right) \supset \Sigma^{\oplus} e_{i} R\left(g_{i}\right) J^{n}$. As a consequence $A=\Sigma^{\oplus}\left(e_{i} R\left(g_{i}\right) \cap A\right)$.

THEOREM 4. Let $R$ be any ring such that $R / J$ is artinian, and let $e$ be a fuxed local idempotent. Then the following are equivalent.

(1) $\left(B_{1}\right)$ holds on the set of local modules of the form $e R / A$, where $e R \supset A \supset e J^{n}$ for some n.

(2) Any two local modules of the form $e R / A$ are mutually almost relative projective, where $A$ is as in (1).

(3) $e R / N$ is uniserial with respect to submodules $A / N$ with $|e R / A|<\infty$ and $(e J)^{2} \subset N$, i.e., any simple sub-factor module $e J^{i} / e J^{i+1}$ of $e R / N$ except $e R / e J$ and $\operatorname{Soc}(e R / N)$ (if it exists) is not isomorphic to $\mathrm{eR} / \mathrm{eJ}$, where $N=\bigcap_{n}\left(e J^{n}\right)$.

Further let $\boldsymbol{M}^{\prime}(e)$ be the set $\{M\}$ given before Theorem 2 such that $M$ is $a$ homomorphic image of $\left(e R / e J^{n}\right)^{(m)}$ for some integers $n$ and $m$. Then the following are equivalent.

(4) $\left(\mathrm{B}_{1}\right)$ holds on $\boldsymbol{M}^{\prime}(e)$.

(5) Any two $R$-modules in $\boldsymbol{M}^{\prime}(e)$ are mutually almost relative projective.

(6) $e R / N$ is unserial with respect to $A / N$ as in (3) and for any $n$ every submodule of $e R \oplus e R$ which contains $e J^{n} \oplus e J^{n}$ is standard.

Proof. (2) $\Rightarrow(1)$. This is trivial.

(1) $\Rightarrow(3)$. Assume $e J^{i} \neq e J^{i+1}$. Let $A$ be a submodule such that $e J^{i+1} \subset A \subset e J^{i}$. Then $e R e A \subset e J^{i}$ and $e J e e R e A \subset e J e e J^{i} \subset e J^{i+1} \subset A$. Hence $e R / A$ and $e R / e R e A$ are mutually almost relative projective by [5, Proposition 2]. Hence $e R / A$ is (almost) $e R / A$-projective by (1), and so $A$ is characteristic. Let $B$ be another submodule such that $e J^{i+1} \subset B \subset e J^{i}$. Then since $A, A+B$ and $B$ are characteristic, $A \subset A+B$ and $e J e(A+B) \subset e J^{i+1} \subset B$, $e R / A$ is (almost) $e R /(A+B)$-projective and $e R /(A+B)$ is almost $e R / B$-projective. Hence $e R / A$ is almost $e R / B$-projective and so $e R / A \oplus e R / B$ has LPSM. Therefore $A \subset B$ or $B \subset A$ by Lemma 1 . Accordingly $e J^{i} / e J^{i+1}$ is simple. Hence $e R / N$ is uniserial with respect to $A / N$. Since $e R / e J^{i}$ is almost $e R / e J^{i+1}$-projective for any $i$, we know that $e R / e J$ is almost $e R / e J^{n}$-projective for all $n$ by $\left(\mathrm{B}_{1}\right)$. Hence $e J e e J \subset e J^{n}$, and so $(e J)^{2} \subset N$.

(3) $\Rightarrow(2)$. This is clear from [5, Proposition 2].

$(5) \Rightarrow(4)$. This is clear.

$(4) \Rightarrow(6)$. We assume $\left(B_{1}\right)$. Then we know from the first equivalence that $e R / N$ is uniserial with respect to $A / N$, and $e R / e J$ is almost $e R / N$-projective by (3), since $N \subset e J$ are characteristic. Moreover $e R / N$ is $M^{\prime}$-projective from [1, p. 22, Exercise 4] for any $M^{\prime}$ in $M^{\prime}(e)$. Hence by $\left(\mathrm{B}_{1}\right) e R / e J$ is almost $M^{\prime}$-projective. Let $e J^{n} \subset A_{i} \subset B_{i}$ be submodules in $e R, i=1,2$ such that $B_{1} / A_{1} \approx B_{2} / A_{2}$ via $g$. First we shall show that $g$ is liftable to an element in $\operatorname{Hom}_{R}\left(e R / e J^{n}, e R / e J^{n}\right.$ ) (cf. the proof (2) $\Rightarrow(1)$ in Theorem 2). If $B_{1}=e R, g$ is easily liftable to an element in $\operatorname{Hom}_{R}\left(e R / e J^{n}, e R / e J^{n}\right)$. Hence we assume $B_{i} \subset e J$. Put $M=(e R \oplus e R) / B_{1}(g) B_{2} \in M^{\prime}(e)$, where $B_{1}(g) B_{2}=\left\{b_{1}+b_{2} \in B_{1} \oplus B_{2} \mid g\left(b_{1}+A_{1}\right)=b_{2}+\right.$ $\left.A_{2}\right\}$. Then $e R / e J$ is almost $M$-projective from the above. Therefore $M$ is decomposable by Lemma 3 and hence $g$ is liftable to an element in $\operatorname{Hom}_{R}\left(e R / A_{1}, e R / A_{2}\right)$ or $\operatorname{Hom}_{R}\left(e R / A_{2}, e R / A_{1}\right)$ by [14, Lemma 2.1] (cf. [7, p. 526, Remark]). As a consequence $g$ is liftable to an element in $\operatorname{Hom}_{R}\left(e R / e J^{n}, e R / e J^{n}\right)$, since $g$ is given by an element in $e R e$. 
Thus every submodule of $e R / e J^{n} \oplus e R / e J^{n}$ is standard by [8, Lemma 5], and we obtain (6) from Lemma 4.

(6) $\Rightarrow(5)$. Assume that $e R / N$ is uniserial as in (6). Then $e R / A_{1}$ is almost $e R / A_{2}$ projective for any $A_{i} \subset e R$ with $\left|e R / A_{i}\right|<\infty$ by (2). Further every module in $M^{\prime}(e)$ is a direct sum of local modules $e R / A$ by $[8$, Lemma 5]. Hence (5) holds by [9, Theorem ] (note that every module in $\boldsymbol{M}^{\prime}(e)$ is of finite length).

Assume that $R$ is semi-perfect and that (1) in Theorem 4 holds for any local modules. Then the above proof $(1) \Rightarrow(3)$ shows that the set of right ideals $\{A \subset e R|| e R / A \mid<\infty\}$ is uniserial. Hence $e R / e J^{i}$ is almost $e R / e J^{j}$-projective for any $i$ and $j$. Further since $e R / e J^{i}$ is (almost) $f R / f J^{i+1}$-projective ( $f$ is a primitive idempotent not isomorphic to $e$ ), any two local modules with finite length are mutually almost relative projective. Hence from [8, Theorem 4] and the proof of Theorem 4 we can get

Proposition 2. Let $R$ be a semi-perfect ring. Then the following are equivalent:

(1) $\left(\mathrm{B}_{1}\right)$ holds for any local modules,

(2) $R$ is a right Nakayama ring with radical square-zero.

Further the following are equivalent:

(3) $\left(\mathrm{B}_{1}\right)$ holds for any finitely generated $R$-modules,

(4) $R$ is a two-sided Nakayama ring with $J^{2}=0$.

4. Transitivity on relative injectives. We shall explore here the dual results to the previous section. We can dually define the concepts of transitivity of (almost) relative injectives and $\left(\mathrm{B}_{1}^{*}\right)$. Let $S$ be a simple $R$-module. By $\boldsymbol{M}(S)$ we denote the set of $R$-modules $M$ such that $\operatorname{Soc}(M) \approx S^{(n(M))}$ with $n(M)<\infty$; by $M^{\prime}(S)$ we denote the set of $M$ in $\boldsymbol{M}(S)$ such that $|M|<\infty$.

Proposition $1^{\#}$. Let $R$ be any ring and $S_{1}$ a simple $R$-module. Then the transitivity of relative injectives on $\boldsymbol{M}\left(S_{1}\right)$ holds. Assume further that $R$ is a right semi-artinian ring. In this case, if the transitivity on $M\left(S_{1}\right) \cup M\left(S_{2}\right)$ holds, then $S_{1}$ and $S_{2}$ are injective, where $S_{2}$ is a simple module not isomorphic to $S_{1}$.

THEOREM $3^{\#}$. Let $R$ be a perfect ring. Then the transitivity of relative injectives over the modules with finite Goldie dimension holds if and only if either $R$ is semi-simple or $R / J(R)$ is a simple ring.

We can obtain the dual result to Proposition 2, which we skip. Finally we observe the dual to Theorem 4.

THEOREM $4^{\#}$. Let $R$ be any ring and $S$ a fuxed simple $R$-module. Then the following are equivalent.

(1) $\left(\mathrm{B}_{1}^{\#}\right)$ holds on the set of all submodules with finite length in $E(S)$.

(2) Any two submodules of finite length in $E(S)$ are mutually almost relative injective.

(3) $E^{\prime}=\bigcup_{n} \operatorname{Soc}_{n}(E)$ is a uniserial module such that any simple sub-factor module of $E^{\prime}$ except $E^{\prime} / J\left(E^{\prime}\right)$ (if it exists) and $\operatorname{Soc}(E)$ is not isomorphic to $\operatorname{Soc}(E)$. $\left(H e r e\left\{\operatorname{Soc}_{n}(E)\right\}\right.$ is the lower Loewy series of $E$.)

Further the following are equivalent.

(4) $\left(\mathrm{B}_{1}^{\#}\right)$ holds on $\boldsymbol{M}^{\prime}(S)$. 
(5) Any two modules in $M^{\prime}(S)$ are mutually almost relative injective.

(6) $E^{\prime}$ is a uniserial module as in (3) and every submodule of $E^{\prime} \oplus E^{\prime}$ is standard.

Proof. First we note that if $\operatorname{Soc}_{n}(E)$ is uniserial for all $n$, and if a submodule $A$ in $E^{\prime}$ is not contained in $\operatorname{Soc}_{n}(E)$ for any $n$, then $A=E^{\prime}$, provided $E^{\prime} \neq \operatorname{Soc}_{t}(E)$ for all $t$. Since $A \notin \operatorname{Soc}_{n}(E)$ for any $n$, there exists $a$ in $A \backslash \operatorname{Soc}_{n}(E)$. $A$ being a submodule in $E^{\prime}, a$ is an element in $\operatorname{Soc}_{m}(E)$ for some $m$, and hence $m>n$. We may assume $a \notin \operatorname{Soc}_{m-1}(E)$. Since $\operatorname{Soc}_{m}(E)$ is uniserial, $\operatorname{Soc}_{n}(E) \subset \operatorname{Soc}_{m}(E)=a R \subset A$. Therefore $A \supset \operatorname{Soc}_{n}(E)$. Now we prove the theorem. This is dual to Theorem 4 . Hence we shall show only (4) $\Rightarrow(6)$. Put $E=E(S)$. Then $E^{\prime}=\bigcup_{n} \operatorname{Soc}_{n}(E)$ is a uniserial module as in (3) from the first equivalence.

Set $E^{*}=E_{1} \oplus E_{2}$ with $E_{i}=E^{\prime}$ and denote the projection of $E^{*}$ onto $E_{i}$ by $\theta_{i}$. Let $B^{*}$ be a submodule of $E^{*}$, and put $B_{i}=\theta_{i}\left(B^{*}\right)$ and $A_{i}=E_{i} \cap B^{*}$. Then $g: B_{1} / A_{1} \approx B_{2} / A_{2}$ and $B^{*}=B_{1}(g) B_{2}$ (cf. [8]). If $A_{1}=0$, then $g$ is liftable to an element $\tilde{g}$ in $\operatorname{End}_{R}\left(E^{\prime}\right)$, since $E^{\prime}$ is characteristic in $E$. Hence $E^{*}=E_{1} \oplus E_{2}(\tilde{g}) \supset 0 \oplus B_{2}(g)=B^{*}$. Therefore we assume $A_{i} \neq 0$ for $i=1,2$. In the dual manner to the proof of Theorem 4, we can show from the first equivalence that $S$ is almost $B^{*}$-injective. On the other hand, since we may assume $A_{i} \neq 0$ and $B_{i} \neq A_{i}, B^{*}$ is decomposable by Lemma $3^{\#}$. Further since $\operatorname{Soc}\left(B^{*}\right)=S \oplus S, B^{*}=$ $D_{1} \oplus D_{2}$ and the $D_{i}$ are isomorphic to submodules in $E^{\prime}$ for the $D_{i}$ are uniform. Hence they are uniserial. Assume $B_{1}=E^{\prime}$ and $\left|E^{\prime}\right|=\infty$. Then $\left|D_{1}\right|=\infty$ or $\left|D_{2}\right|=\infty$. We may assume that $\left|D_{1}\right|=\infty$. Since $D_{1}$ is uniform, $\theta_{i} \mid D_{1}$ is a monomorphism for $i=1$ or 2 , say $i=1$. Since $\left|D_{1}\right|=\infty, \theta_{1} \mid D_{1}$ is an isomorphism from the initial remark. Putting $h=$ $\theta_{2}\left(\theta_{1} \mid E^{\prime}\right)^{-1}: E^{\prime} \rightarrow E^{\prime}$, we obtain $E^{*}=E^{\prime}(h) \oplus E^{\prime}$ and $D_{1}=E^{\prime}(h) \subset B^{*}$. Hence $B^{*}=$ $E^{\prime}(h) \oplus B^{*} \cap E^{\prime}$ and $B^{*} \cap E^{\prime} \subset E^{\prime}$. Therefore $B^{*}$ is standard. Finally we assume $\left|B_{i}\right|<\infty$ for $i=1,2$. Then $B^{\prime}=B \oplus B_{2} \supset B^{*}$. Let $j_{i}$ be the projection of $B^{\prime}$ onto $B_{i}$. We may suppose $\left|B_{1}\right| \geqq\left|B_{2}\right|$. Since $B^{*}=B_{1}(g) B_{2}, \pi_{1}\left(B^{*}\right)=B_{1}$, and hence we assume $\pi_{1}\left(D_{1}\right)=B_{1}$. On the other hand, since $D_{1}$ is uniform, $D_{1}$ is monomorphic to a submodule of $B_{1}$ or $B_{2}$, i.e. $\left|D_{1}\right| \leqq\left|B_{1}\right|$. Hence $\pi_{1} \mid D_{1}$ is an isomorphism. Put $h=\pi_{2} \pi_{1}^{-1} \mid B_{1}: B_{1} \rightarrow B_{2}$. Then $B^{\prime}=B_{1}(h) \oplus B_{2}$ and $B_{1}(h)=D_{1}$. As a consequence $B^{*}=B_{1}(h) \oplus B^{*} \cap B_{2}$. Since $E$ is injective and $E^{\prime}$ is characteristic, we obtain an extension $h^{\prime}$ of $h$ in $\operatorname{End}_{R}\left(E^{\prime}\right)$. Hence $E^{*}=E_{1}\left(h^{\prime}\right) \oplus E_{2} \supset B_{1}\left(h^{\prime}\right) \oplus B^{*} \cap E_{2} \supset B_{1}(h) \oplus B^{*} \cap B_{2}=B^{*}$, i.e. $B^{*}$ is standard in $E^{*}$.

\section{REFERENCES}

1. T. Albu and C. Nastasescu, Relative finiteness in module theory, Monographs and Textbooks in Pure and Applied Mathematics 84 (Marcel Dekker, 1984).

2. G. Azumaya, F. Mbuntum and K. Varadarajan, On $M$-projective and $M$-injective modules, Pacific J. Math. 59 (1975), 9-16. $115-135$.

3. K. R. Fuller, On indecomposable injectives over artinian rings, Pacific J. Math. 29 (1969),

4. Y. Baba, Note on almost $M$-injectives, Osaka J. Math. 26 (1989), 687-698.

5. Y. Baba and M. Harada, On almost relative projectives and injectives, Tsukuba Math. J. 14 (1990), 53-69.

6. M. Harada, Uniserial rings and lifting properties, Osaka J. Math. 19 (1982), 217-229.

7. M. Harada, Generalization of Nakayama ring III, Osaka J. Math. 23 (1986), 523-539.

8. M. Harada and A. Tozaki, Almost $M$-projectives and Nakayama rings, J. Algebra 122 (1989), 447-474.

9. M. Harada, On almost relative projectives over perfect rings, Osaka J. Math. 27 (1990), $465-482$. 
10. M. Harada, On almost relative injectives on artinian modules, Osaka J. Math. 27 (1990), 963-971.

11. M. Harada and T. Mabuchi, On almost $M$-projectives, Osaka J. Math. 26 (1989), 837-848.

12. M. Harada, Hereditary rings and almost relative projectives, to appear.

13. K. Oshiro, Semiperfect modules and quasi-semiperfect modules, Osaka J. Math. 20 (1983), 337-373.

14. T. Sumioka, Tachikawa's theorem on algebras of left colocal type, Osaka J. Math. 21 (1984), 629-648.

15. T. Sumioka, On artinian ring of right local type, Math. J. Okayama Univ. 29 (1987), $127-146$.

16. R. Wisbauer, Grundlagen der Modul- und Ringtheorie. Ein Handbuch für Studium and Forschung (Reinhard Fischer, 1988).

Department of Mathematics

Osaka City University

SUGIMOTO-3, SUMIYOSI-KU

OSAKA 558

JAPAN 